\title{
India Ink Incorporated Multifunctional Phase-transition Nanodroplets for Photoacoustic/Ultrasound Dual-modality Imaging and Photoacoustic Effect Based Tumor Therapy
}

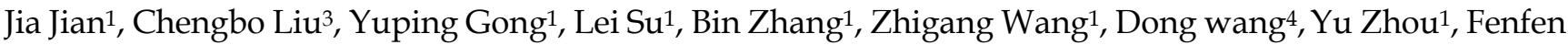 \\ $\mathrm{Xu}^{1}$, Pan Li ${ }^{1}$, Yuanyi Zheng ${ }^{1}$, Liang Song ${ }^{3 凶}$, Xiyuan Zhou ${ }^{2 \bowtie}$ \\ 1. Institute of Ultrasound Imaging, The Second Affiliated Hospital of Chongqing Medical University, Chongqing 400010, China. \\ 2. Department of Ophthalmology, The Second Affiliated Hospital of Chongqing Medical University, Chongqing 400010, China. \\ 3. Research Lab for Biomedical Optics and Molecular Imaging, Shenzhen Key Lab for Molecular Imaging, Institute of Biomedical and Health En- \\ gineering, Shenzhen Institutes of Advanced Technology, Chinese Academy of Sciences, Shenzhen 518055, China. \\ 4. Department of Ultrasound, The Children's Hospital of Chongqing Medical University, Chongqing 400014, China.
}

\begin{abstract}
$\triangle$ Corresponding author: Xiyuan Zhou, Tel./fax: +86 23 63693589; E-mail: cb.liu@siat.ac.cn. Or Liang Song, Tel: +86 755 86392240; E-mail: liang.song@siat.ac.cn.

(c) Ivyspring International Publisher. This is an open-access article distributed under the terms of the Creative Commons License (http://creativecommons.org/ licenses/by-nc-nd/3.0/). Reproduction is permitted for personal, noncommercial use, provided that the article is in whole, unmodified, and properly cited.
\end{abstract}

Received: 2014.05.28; Accepted: 2014.07.10; Published: 2014.08.0I

\begin{abstract}
The in vivo applications of gas-core microbubbles have been limited by gas diffusion, rapid body clearance, and poor vascular permeability. To overcome these limitations, using a modified three-step emulsion process, we have developed a first-of-its-kind India ink incorporated optically-triggerable phase-transition perfluorocarbon nanodroplets (INDs) that can provide not only three types of contrast mechanisms - conventional/thermoelastic photoacoustic, phase-transition/ nonlinear photoacoustic, and ultrasound imaging contrasts, but also a new avenue for photoacoustic effect mediated tumor therapy. Upon pulsed laser illumination above a relatively low energy threshold, liquid-gas phase transition of the INDs has been demonstrated both in vitro and in vivo, offering excellent contrasts for photoacoustic and ultrasound dual-modality imaging. With further increased laser energy, the nanodroplets have been shown to be capable of destructing cancer cells in vivo, presumably due to the photoacoustic effect induced shock-wave generation from the carbon particles of the incorporated India ink. The demonstrated results suggest that the developed multifunctional phase-transition nanodroplets have a great potential for many theranostic biomedical applications, including photoacoustic/ultrasound dual-modality molecular imaging and targeted, localized cancer therapy.
\end{abstract}

Key words: India Ink, nanodroplets

\section{Introduction}

Microbubbles with gas cores have been widely explored in the field of diagnostic ultrasound over the past few decades. ${ }^{1,2}$ However, the in vivo applications of microbubbles are limited by their short circulation lifetime owing to gas diffusion and biological clearance, as well as poor vascular permeability due to their micrometer-scale dimensions (which prevents them from extravasating out of the vascular system into the tumor intercellular space efficiently). As a result, microbubble-assisted tumor imaging, molecular diagnosis, and therapy have been confined to limited applications. It is believed that only particles with sizes less than a few hundred nanometers can permeate the leaky tumor vasculature. Therefore, exogenous 
contrast agents smaller than such sizes should be synthesized to optimize in vivo applications.,4 Phase-transition perfluorocarbon (PFC) liquid nanodroplets have been gaining more and more attention in recent years. 5,6 These nano-sized droplets can efficiently permeate out of the blood circulation into tumor tissues. Although their liquid cores initially provide poor ultrasound contrast, ${ }^{7}$ once triggered into gaseous phase, they can offer significantly augmented ultrasound contrast. Compared with microbubbles, PFC liquid nanodroplets, which are further stabilized by lipids, polymers, surfactants, or albumin shells, possess longer circulation lifetime. Traditionally, ultrasound irradiation with a relatively high pressure amplitude was used to trigger the PFC liquid into gaseous phase-also termed as acoustic droplet vaporization (ADV). ${ }^{6}$ However, the Laplace pressure and the surface-stabilizing agents of the droplets make them relatively difficult to vaporize by ultrasound. The pressures and frequencies of conventional diagnostic ultrasound are proved not sufficient to induce acoustic droplet vaporization. 8,9 In fact, acoustic droplet vaporization typically requires acoustic frequencies and pressures that may also cause unwanted bioeffects, ${ }^{9}$ which is certainly undesirable.

Recently, the development of optical droplet vaporization (ODV) has provided an alternative way to activate PFC nanodroplets. ${ }^{10,11}$ By incorporating optical absorbing materials (i.e., optical absorbers) into the liquid cores, laser irradiation instead of ultrasound (US) exposure has been applied to induce the liquid-to-gas transition of PFC nanodroplets. When relatively low pulsed laser energy is used, conventional photoacoustic (PA) signals originating from the transient thermoelastic expansion of the optical absorbers are generated. However, if the laser energy goes up beyond a certain threshold, the absorbed optical energy will lead to a local temperature rise sufficient to induce the liquid-to-gas transition (vaporization) of the droplets, which, in turn, can trigger the generation of much stronger (nonlinear) temporary photoacoustic signals compared with those from thermoelastic expansion. ${ }^{11-14}$ Furthermore, once triggered into gas phase, such nanodroplets can provide excellent contrast for ultrasound imaging as well. ${ }^{15}$

Photoacoustic imaging is a hybrid biomedical imaging modality that possesses the contrast superiority of optical imaging and the resolution superiority and penetration ability of ultrasound imaging. ${ }^{16-22}$ The photoacoustic effect converts the absorbed pulsed optical energy into acoustic waves, which are detected by ultrasound transducers to reconstruct the optical absorption distribution in biological tissue. ${ }^{23}$ In order to obtain high PA contrast from exogenous agents, PA signals from endogenous tissue chromophores should be minimized. It is well known that the optical absorption of major biological tissue chromophores (e.g., hemoglobin) is minimal in the near-infrared (NIR) region from 700 to $1100 \mathrm{~nm}$. Moreover, due to significantly reduced optical scattering, the optical penetration depth of tissue is relatively large in this wavelength range. Therefore, it is of great significance to develop contrast agents with peak absorption in this wavelength range (e.g., PFC naodroplets incorporating NIR optical absorbers). ${ }^{24}$

By now, a number of different optical absorbers have been used for ODV materials, including $\mathrm{PbS}$ nanoparticles, plasmonic gold nanoparticles (rods and spheres), and ICG.10,11,25,26 All of them have demonstrated great potential for a variety of photoacoustic and ultrasound imaging applications. However, PbS based nanodroplets have been shown to require a very high laser fluence (on the order of $\mathrm{J} / \mathrm{cm}^{2}$ ) to induce the liquid-to-gas transition, ${ }^{10}$ which may cause potential damage to biological tissue as well. Plasmonic gold nanoparticles may get melted easily under moderate laser illumination, ${ }^{11}$ which may shift the absorption spectrum to undesired wavelengths. ICG is a FDA approved agent used in the clinic, however, compared with India ink, ICG has a much lower molar extinction coefficient (for example, more than 10 times lower at $800 \mathrm{~nm}$ ) and a narrower optical absorption spectrum. ${ }^{27,28}$ In addition, for PA applications, ICG also suffers from relatively low PA conversion efficiency due to the conversion of fluorescence. Furthermore, all currently reported nanodroplets require laser illumination within a relatively narrow spectral band that may limit the choices of lasers and thus their applications under certain circumstances. In this work, a novel India ink incorporated optically-triggerable phase-transition nanodroplets have been developed for both biomedical imaging and therapy applications. As the optical absorbers of the droplets, India ink is chemically and spectroscopically stable, easily accessible, inexpensive, nontoxic, and have been widely used in the clinic. ${ }^{29-33}$ In addition, the ink's "active constituent" carbon black is nonreactive and nonallergenic; its absorption coefficient is large, with relatively small variations across a wide range of the visible and NIR regions. Thus, over a broad spectrum, it can offer excellent photoacoustic contrast and be optically vaporized. ${ }^{34}$ In addition, shock wave generation from carbon materials upon pulsed laser illumination has been recently reported to offer a potentially new therapeutic mechanism to destruct cancer cells. ${ }^{35-37}$ Therefore, incorporating India ink, and thus this mechanism, into the optically-triggerable phase-transition nanodroplets may of- 
fer a new avenue for targeted and localized cancer therapy.

A typical ink nanodroplet (IND) reported in this work consists of a droplet of liquid Perfluorohexane (PFH) and ink particles within a poly-lactic-coglycolic acid (PLGA) shell. PFH with a boiling point of $56^{\circ} \mathrm{C}$ is stable at room temperature and can be triggered to gaseous phase using proper laser energy. ${ }^{38}$ The polymer PLGA is FDA approved and has been clinically used in biological and medical applications. As the carrier of the droplet, it is highly stable; it also has good biocompatibility, long blood circulation time, and good acoustic properties, without immunogenicity and potential risk for cross infection. ${ }^{39,40}$ The INDs were synthesized using a modified three-step emulsion process. After fabrication, the INDs were characterized by scanning electron microscopy, spectrophotometry, and a laser particle size analyzer. Upon illumination with proper laser energy, the phase-transition process of the INDs was successfully demonstrated in vitro and in vivo, providing excellent contrast for both PA and US imaging. Furthermore, the feasibility of tumor therapy in vivo using the INDs was also demonstrated and studied.

\section{Materials and methods}

\section{Materials}

India ink was purchased from Amresco (USA), PFH was purchased from Alfa Aesar (U.K.), PLGA (50:50, 12,000 Da MW) was purchased from Daigang (China), polyvinyl alcohol (PVA 25,000 Da MW) was obtained from Sigma-Aldrich (USA), methylene chloride $\left(\mathrm{CH}_{2} \mathrm{Cl}_{2}\right)$ was purchased from Chuandong (China), 3-(4,5-dimethylthiazol-2-yl)-2,5-diphenyltetrazoliumbromide (MTT) was obtained from Beyotime (China), Agarose was purchased from Invitrogen (USA). Deionized water was obtained with the Milli-Q Plus System (Millipore Corporation, USA).

\section{Synthesis of INDs}

INDs encapsulating India ink were fabricated with an modified three-step emulsion process. Four solutions were prepared: (1) $4 \mathrm{~mL} 2.5 \% \mathrm{w} / \mathrm{v}$ PLGA solution of $\mathrm{CH}_{2} \mathrm{Cl}_{2}$, (2) $20 \mathrm{~mL} \mathrm{4 \%} \mathrm{w/v} \mathrm{PVA} \mathrm{aqueous}$ solution, (3) $0.5 \mathrm{~mL} 10 \% \mathrm{v} / \mathrm{v}$ India ink aqueous solution, and (4) $0.3 \mathrm{~mL}$ PFH. First, the $0.5 \mathrm{~mL}$ ink aqueous solution was added to the $0.3 \mathrm{~mL}$ PFH and emulsified in an ice bath with an ultrasonic probe (SONICS \& MATERALS Inc; USA) at $130 \mathrm{~W}$ for $1 \mathrm{~min}$. After that, $4 \mathrm{~mL} 2.5 \% \mathrm{w} / \mathrm{v}$ PLGA solution of $\mathrm{CH}_{2} \mathrm{Cl}_{2}$ was added to the emulsion and emulsified in the ice bath with the ultrasonic probe at $130 \mathrm{~W}$ for another $2 \mathrm{~min}$, and then $20 \mathrm{~mL} \mathrm{4 \%} \mathrm{w/v} \mathrm{PVA} \mathrm{aqueous} \mathrm{solution} \mathrm{was} \mathrm{added} \mathrm{to}$ the double emulsion and homogenized (FJ300-SH, China) in an ice bath within $3 \mathrm{~min}$ for the third emul- sion. Next, the emulsion was stirred for 2 hours by a magnetic stirrer (HJ-1, Ronghua, China), and then centrifuged (Eppendorf AG, Germany) at $8000 \mathrm{rpm}$ for 5 minutes. After centrifugation, the supernatant was discarded, and the droplet precipitate was washed by deionized water. Both the centrifugation and washing process were repeated for three times. Finally, the washed spheres were diluted in $2.5 \mathrm{~mL}$ degassed deionized water to prepare the stock with the concentration of $40 \mathrm{mg} / \mathrm{mL}$ and then stored in a centrifuge tube at $4{ }^{\circ} \mathrm{C}$ for further use. It is to be noted that both stock and diluted India ink was pretreated with ultrasonic sonication (immersed in ultrasonic water bath (SCQ-H500, Shengyan, China) for about 30 minutes ${ }^{29}$ ) before usage throughout the whole experiment.

\section{Characterization of INDs}

A UV/VIS/NIR Spectrophotometer (UV3600; Shimadzu, Japan) was used to acquire the optical absorption spectra (optical density vs. wavelength) of the INDs and the ink alone. The morphological and structural characterization and size of INDs were observed by a scanning electron microscope (SEM, FEI Nova NanoSEM450; USA) and an optical microscope (Olympus CKX41; CANADA). The size distribution and zeta potential of INDs were measured by a Laser Particle Size Analyzer System (Zeta SIZER3000HS; Malvern, USA).

\section{Cell culture}

MDA-MB-231 human breast cancer cells were obtained from the laboratory of Ultrasound Engineering Institute of Chongqing Medical University and cultured using RPMI 1640 with 10\% fetal bovine serum, and $1 \%$ penicillin/ streptomycin at $37^{\circ} \mathrm{C}$ with humidified air containing $5 \% \mathrm{CO}_{2}$.

\section{Animal models}

All animals were treated according to the guidelines of the Care and Use of Laboratory Animals. Female BALB/c nude mice (4-6 weeks old and weighted 18-20 g) were purchased from Slac Laboratory Animal Co. Ltd (Shanghai, China). For inoculation, MDA-MB-231 cells $\left(1 \times 10^{6}\right.$ cells $/ 100 \mu \mathrm{L} /$ mouse $)$ were suspended in serum-free RPMI-1640 medium and administered subcutaneously to the flanks of the mice. Tumor-bearing mice were used for experiments 7 to 10 days after tumor inoculation when the volume $\left[\pi / 6 \times\right.$ length $\times$ (breadth) $\left.{ }^{2}\right]$ reached about $200 \mathrm{~mm}^{3}$.

\section{Cytotoxicity study}

INDs $(5 \mathrm{~mL})$ were sterilized under UV light for 20 min. MDA-MB-231 cells were incubated in cell-culturing media containing INDs at concentrations of $0.3,0.6,0.9,1.2$, and $1.5 \mathrm{mg} / \mathrm{mL}$. The cells 
were first seeded into 96-well plates at a density of $5 \times 10^{3}$ cells per well and incubated at $37^{\circ} \mathrm{C}$ and $5 \% \mathrm{CO}_{2}$ for 12 hours. Then the former medium was replaced with new medium containing varying concentrations of INDs, and the cells were incubated at $37^{\circ} \mathrm{C}$ and $5 \%$ $\mathrm{CO}_{2}$ for 24 hours. The cells in the culture media without any INDs were regarded as the blank control. Afterwards, $150 \mu \mathrm{L}$ new media and $20 \mu \mathrm{L}$ MTT solution were added to each well to replace the former medium, and incubated at $37^{\circ} \mathrm{C}$ and $5 \% \mathrm{CO}_{2}$ for 4 hours. Then the former medium was abandoned and $100 \mu \mathrm{L}$ DMSO was added to each well. Low speed oscillation was applied for 10 minutes to make sure the formazan crystals were dissolved completely, and the optical absorbance was then measured at $490 \mathrm{~nm}$ on a microplate reader (Tecan Sunrise, Switzerland). ${ }^{41}$ The results were expressed as percentages relative to those acquired in the blank control. The absorbance differences between INDs groups and the control group were analyzed statistically using analysis of variance. The differences observed between samples of varying concentrations were considered significant for $\mathrm{P}<0.05$.

\section{Instrumentation for PA and US measurement}

A custom-made photoacoustic and ultrasound dual-modality imaging system was used for all the PA measurements. The system consists of a tunable pulsed OPO laser (mostly operating at $\sim 800 \mathrm{~nm}$ ) to illuminate the objects through a custom-built light delivery system, a $10-\mathrm{MHz}$ focused ultrasound transducer for both US and PA signal acquisitions, and a precision 3D motorized stage to move the imaging head across the $x-y$ plane for 3D imaging - as the depth resolution comes from the time of arrival of the received acoustic signals, the stage's $z$ axis is used for positioning the imaging head only. More details of the imaging system can be found in our earlier publication. ${ }^{42}$ In general, depth resolved PA and US signals were acquired sequentially, and a 3D image of the object was obtained with 2D raster scanning of the imaging head in the $x-y$ plane.

A Q-switched Nd:YAG pulsed laser (532 nm, 5 $\mathrm{Hz}$ repetition rate, $1200 \mathrm{~mJ}$ maximum pulse energy, 10 ns pulse width) was applied for INDs phase transition triggering in all experiments without PA measurements, after which a commercial ultrasonic diagnostic instrument (Mylab90, Esaote, Italy) with an US frequency of $10 \mathrm{MHz}$ and an imaging speed of 50 frames/s was used to acquire US images in a routine mode (B-mode) and contrast mode (mechanical index $(\mathrm{MI})=0.1)$. Compared with the PA/US dual-modality system mentioned above, the laser source here supplies a much higher maximum single pulse energy (1200 mJ/pulse vs. $1 \mathrm{~mJ} /$ pulse) while the ultrasonic system can offer an imaging speed more than 100 times faster.

\section{In vitro INDs characterization and imaging}

PA signals of 4 INDs concentrations (5, 10, 20, 40 $\mathrm{mg} / \mathrm{mL}$ ) in $2 \%$ agarose gel phantom were first measured to characterize the photoacoustic linearity of the nanodroplets $(800 \mathrm{~nm}$, OPO laser, laser fluence is 0.5 $\mathrm{mJ} / \mathrm{cm}^{2}$ ). It was assumed the number of synthesized nanodroplets was linearly related to how much PLGA was used, and therefore the concentration of the droplets was indicated by the PLGA quantity (e.g. 40 $\mathrm{mg} / \mathrm{mL}$ ) in this manuscript. Furthermore, the following procedures were carried out to estimate how much ink particles were incorporated into each nanodroplet. The optical density of INDs was compared with that of pure PLGA droplet (without any incorporated ink) of the same concentration to estimate the absorption of ink particles encapsulated in INDs using spectrophotometry. Afterwards stock ink particles were titrated into degassed deionized water until the optical density matched that of the INDs incorporated ink particles just obtained. ${ }^{11}$

To compare the photoacoustic property difference between INDs and pure ink, both INDs and ink solution containing the same amount of ink particles were irradiated by the laser source of our PA system ( $800 \mathrm{~nm}$, OPO laser, laser fluence is $8 \mathrm{~mJ} / \mathrm{cm}^{2}$ ) for 100 seconds (1000 laser pulses), while photoacoustic signals were recorded over time. Moreover, to validate nanodroplet vaporization, INDs, pure ink (same ink particle concentration as that incorporated in INDs) and pure droplet (without any ink incorporated, same PLGA concentration as INDs) solution were observed with US imaging before and after laser irradiation (532 nm, Nd:YAG laser, $10 \mathrm{~s}$ irradiation, laser fluence is $5 \mathrm{~mJ} / \mathrm{cm}^{2}$ ).

\section{In vivo INDs study}

\section{In vivo INDs imaging}

All in vivo imaging experiments were conducted with laser energy density between $0.5-12 \mathrm{~mJ} / \mathrm{cm}^{2}$, well below the ANSI laser safety limit. ${ }^{43}$ The animals were anesthetized via Isoflurane breathing (1.5\% Isoflurane gas mixed with oxygen) throughout the course of the measurement. First of all, to validate the contrast enhancement for conventional photoacoustic imaging, photoacoustic images of the tumor areas were acquired in situ before and after intratumoral injection of INDs $(50 \mu \mathrm{L}$ INDs, $40 \mathrm{mg} / \mathrm{mL}, 800 \mathrm{~nm}$, OPO laser, laser fluence is $0.5 \mathrm{~mJ} / \mathrm{cm}^{2}$, tumor massaged for $1 \mathrm{~min}$ after injection). Furthermore, to validate the in vivo nanodroplet vaporization, US images of the tumor area injected with INDs, pure ink (same ink particle concentration as that incorporated in 
INDs), and pure droplets (without any ink incorporated, same PLGA concentration as INDs) were obtained before and after laser irradiation (532 nm, $\mathrm{Nd}$ :YAG laser, $10 \mathrm{~s}$ irradiation, laser fluence is 12 $\mathrm{mJ} / \mathrm{cm}^{2}$ ). In total, 30 mice were used for experiments. Among them, 15 mice were used in the PA imaging group with intratumoral injection of INDs, pure ink, and pure droplets ( 5 mouse each group); the other 15 mice were also divided into three sub-groups, with 5 in each group injected intratumorally in situ with INDs, pure ink, and pure droplets, respectively.

\section{In vivo INDs therapy}

To study the therapy effect of INDs, 10 tumor-bearing mice were randomly divided into two groups ( $\mathrm{n}=5$ per group): INDs group (I) and INDs blank group (II) as control. In the INDs group, the mice first received in situ intratumoral injection of 50 $\mu \mathrm{L}$ INDs and massaged for $1 \mathrm{~min}$ while in the control group, same amount of saline was injected as sham treatment. The tumors in both groups were then irradiated with laser energy for $3 \mathrm{~min}$ (532 nm, Nd:YAG laser, laser fluence is $18 \mathrm{~mJ} / \mathrm{cm}^{2}$ ). The laser spot was adjusted to cover the whole tumor area.

\section{Evaluation of INDs impact on tumor after laser exposure}

\section{INDs impact on tumor after in vivo imaging}

Six hours after INDs based PA and US imaging in vivo, the animals were euthanized and tumors were removed for macroscopic and microscopic examinations to verify if any side effect exists due to laser illumination during the imaging process. $1 \mathrm{~cm}^{3}$ tissue containing INDs injected area and surrounding area from each tumor was excised, sectioned and stained with hematoxylin and eosin (HE) for pathological examination. Another $1 \mathrm{~cm}^{3}$ tissue was sampled by glutaraldehyde to observe the ultra-structure changes of the targeted tissue with transmission electron microscope (TEM, Hitachi, 7500; Japan). Immunohistochemical examination was also performed to acquire the information on apoptosis of the tumor tissues using terminal dexynucleotidyl transferase(TdT)-mediated dUTP nick end labeling (TUNEL). The nucleus of apoptosis-positive cells would appear in brown color. The proportion of the positive cells, also called apoptosis index (AI), was calculated via reading 5 random regions of the sample at $400 \times$ magnification.

\section{Tumor therapy effect of INDs}

The results of INDs therapy study were evaluated with the same methods as that in the previous in vivo imaging part.

\section{Statistical analysis}

For statistical analysis, each experiment was performed at least three times. One-way analysis of variance was carried out to calculate the differences among each group. Data was presented as mean \pm standard deviation.

\section{Results and Discussion}

\section{Synthesis and characterization of INDs}

Fig. 1A (a-d) shows the fabrication process of INDs: a) PFH was embedded with ink particles suspended in water as the first emulsion; b) PFH and ink particles was encapsulated by PLGA dissolved in $\mathrm{CH}_{2} \mathrm{Cl}_{2}$ as the second emulsion; c) PFH, ink and PLGA was encapsulated by PVA as the third emulsion; $\mathrm{d}$ ) $\mathrm{CH}_{2} \mathrm{Cl}_{2}$ on PLGA shell evaporated by magnetic stirring. Fig. 1A (e) shows the phase transformation process of INDs after laser irradiation. As time goes on, some portion of the initially generated gas leaked out of the PLGA shell, forming small nanobubbles. These nanobubbles can further fuse into micrometer-sized bubbles. Fig. 1B shows the cross-section of the INDs. The porous PLGA shell encapsulates India ink and PFH inside, forming a stable spherical droplet.

Several approaches were used to characterize the INDs. First of all, the fabricated INDs in the emulsion were reserved and monitored for two weeks. No obvious shape and size changes were observed, demonstrating their high stability. Fig. $2 \mathrm{a}_{1}$ shows the SEM image of INDs sample, from which it can be seen that the majority of the INDs presented have an almost perfectly spherical shape. Fig. $2 \mathrm{a}_{2}$ and $2 \mathrm{a}_{3}$ show the SEM images of a single spherical and hemispheric IND, respectively. Porous surface can be seen on most INDs - pores appear as black spots on the surface and were indicated by the white arrows in Fig. $2 \mathrm{a}_{2}$. In Fig. $2 \mathrm{a}_{3}$, ink particles encapsulated within the PLGA shell can be seen through the expanded surface pores (indicated by the white arrows). The porous surface feature of INDs as well as the encapsulated ink particles suggested its potential capability of chemotherapeutic drug delivery for cancer treatment. Moreover, the INDs may enable controlled drug release. While the surface pores of PLGA shell allow drugs to be released slowly, ${ }^{44-46}$ the phase-transition process of INDs may lead the drugs to be released more quickly, due to the expansion or rupture of the PLGA shell. Fig. 2C-2E show the optical density of INDs, size distribution, and zeta potential, measured by spectrophotometry, dynamic light scattering (DLS), and electrophoretic light scattering(ELS), respectively. It can be seen that the absorption spectra of INDs and pure India ink are very similar to each other due to negligible absorption of pure droplet, demonstrating 
the optical property was not significantly altered during the INDs synthesis process. INDs exhibited narrow size distribution with an average hydrodynamic diameter of $472.5 \pm 78.01 \mathrm{~nm}$. The average zeta potential is $50.5 \pm 4.89 \mathrm{mV}$, indicating the high stability of INDs.

Fig. 3A and 3B show the shape and size characteristics of INDs under optical microscope (Olympus
CKX41, CANADA) before and after laser irradiation (532 nm, Nd:YAG laser, laser fluence is $5 \mathrm{~mJ} / \mathrm{cm}^{2}$ ). It was shown that after laser irradiation, gas bubbles were formed due to INDs vaporization (indicated by black arrows in Fig. 3B), which were produced by the escaping of the gas from the PLGA shell. The larger bubbles are presumably due to rapid coalescence of smaller bubbles.

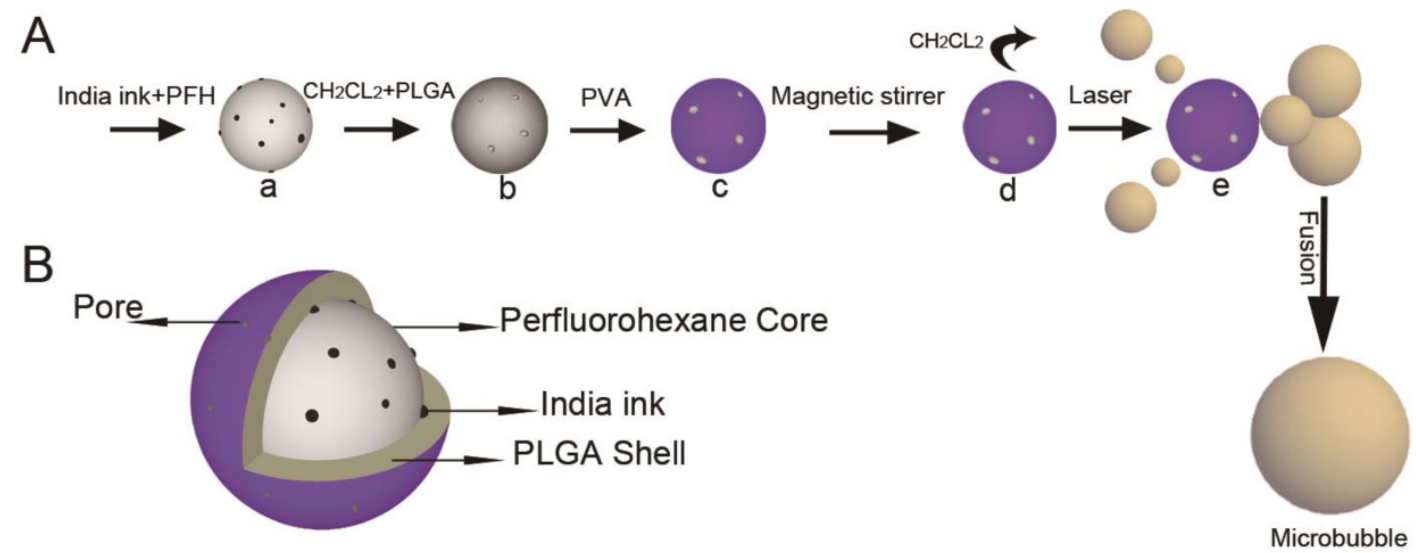

Figure I. Schematic illustration of $(A)$ the IND fabrication process $(a-d)$ and ODV principles $(d$ and e), as well as (B) the architecture of a synthesized IND.

A
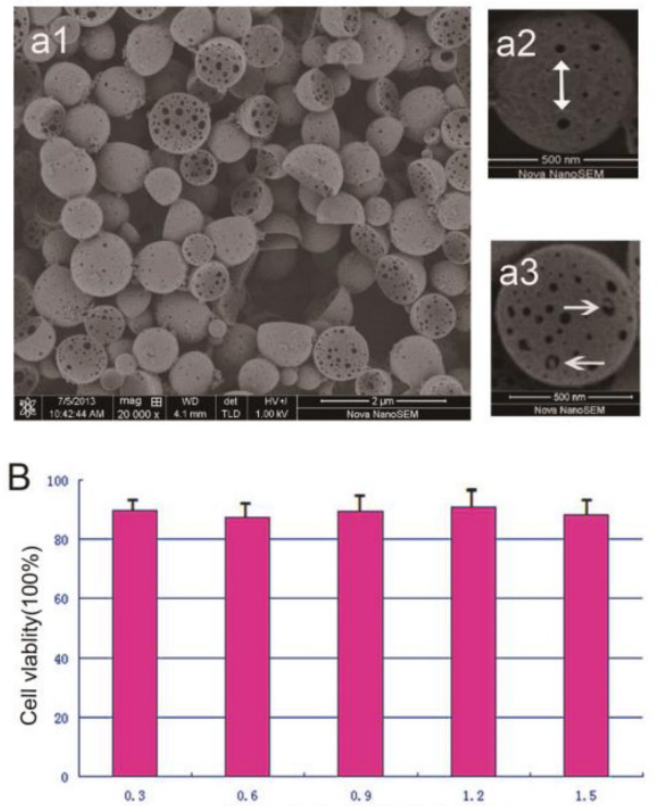

C

Concentration of INDs $(\mathrm{mg} / \mathrm{mL})$

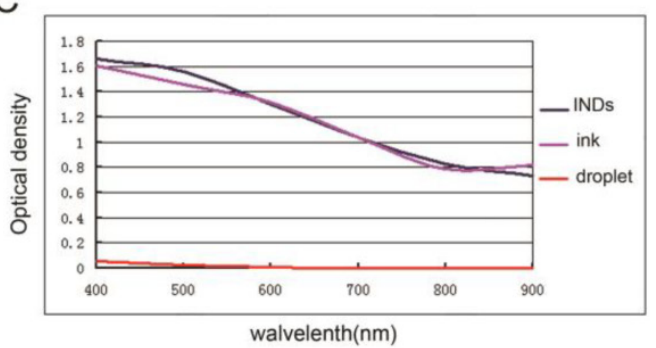

$\mathrm{D}$

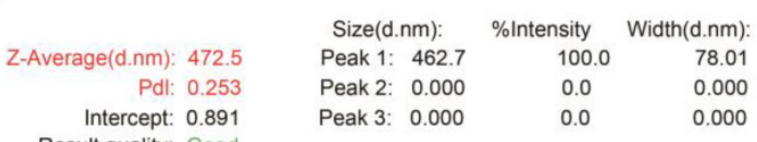

Result quality: Good

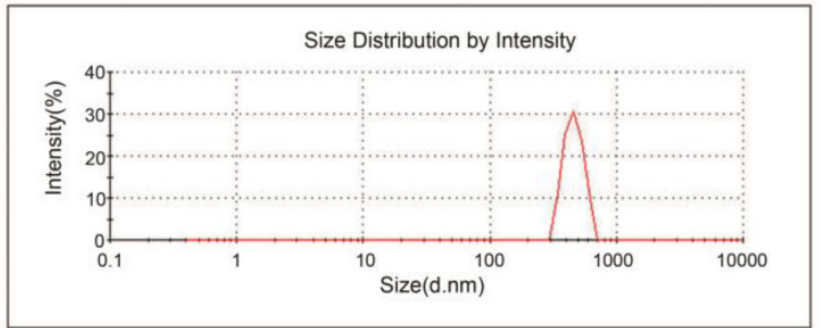

E

Zeta Potential $(\mathrm{mV}) \cdot-50.5 \quad M e a n(\mathrm{mV}) \quad$ Area(\%) Width $(\mathrm{mV})$

Zeta Deviation(mV): 4.89 Conductivity $(\mathrm{mS} / \mathrm{cm}): 0.00106$ Result quality: Good

$\begin{array}{lll}\text { Peak 2: } 0.00 & 0.0 & 0.00\end{array}$

$\begin{array}{lll}\text { Peak 3: } 0.00 \quad 0.0 & 0.00\end{array}$

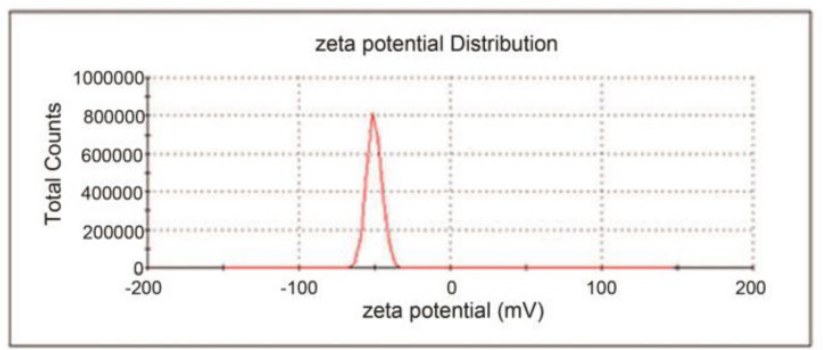

Figure 2. A, SEM images of INDs at different magnifications. Porous surface was indicated by the white arrow in $\mathrm{a}_{2}$, and ink particles encapsulated inside were indicated in a3. B, Cytotoxicity results of INDs on MDA-MB-23I cells (meantstandard deviation for different concentrations of INDs). C-E, The optical density, size distribution, and zeta potential of INDs measured by spectrophotometry, dynamic light scattering (DLS), and electrophoretic light scattering(ELS), respectively. 

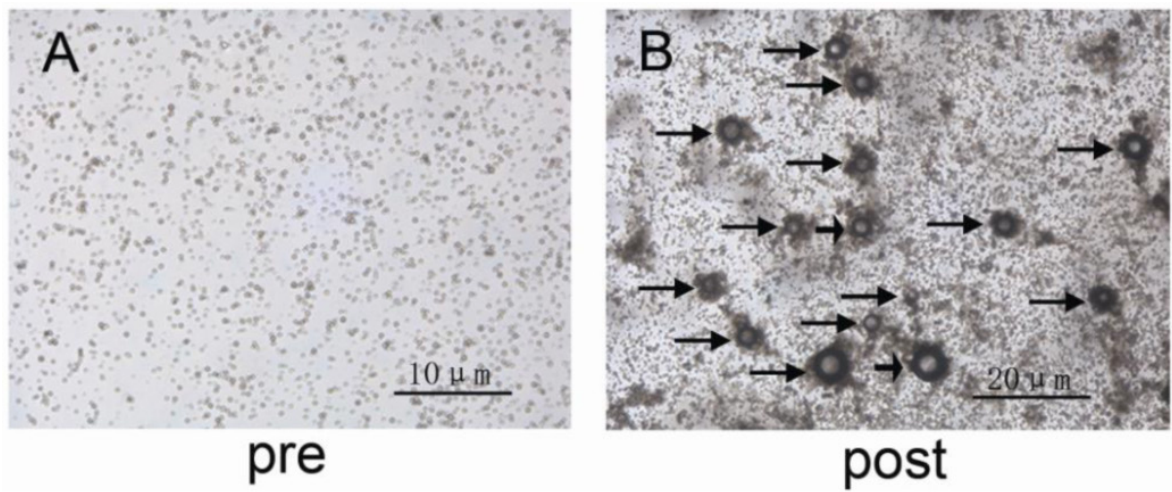

Figure 3. A and B, Bright-field optical microscopic images of the INDs sample pre and post laser exposure, respectively. The black arrows in B indicate the formed bubbles due to INDs vaporization. The larger bubbles are due to rapid coalescence of smaller bubbles.

\section{Cytotoxicity}

The MDA-MB-231 cell viability was measured to be $89.6 \%, 87.5 \%, 89.5 \%$, $90.8 \%$, and $88.3 \%$, (mean \pm standard deviation given in Fig. 2B) for groups cultured with INDs at concentration of $0.3,0.6,0.9,1.2$ and $1.5 \mathrm{mg} / \mathrm{mL}$ respectively. These results neither showed a definite correlation between INDs concentration and cell viability, nor did they show a significant difference among INDs groups compared with the control group $(P>0.05)$, suggesting that INDs has very low or no toxicity to MDA-MB-231 cell line.

\section{In vitro INDs characterization and imaging}

Fig. 4A and 4B show the PA signal dynamics of INDs and ink solution during laser irradiation for 100 $\mathrm{s}\left(800 \mathrm{~nm}\right.$, OPO laser, laser fluence is $\left.8 \mathrm{~mJ} / \mathrm{cm}^{2}\right)$. For INDs, PA signal first goes up immediately and then declined to a lower steady state after about $30 \mathrm{~s}$. The instant increase of the photoacoustic signals (which can be up to 10 times larger than the conventional photoacoustic signals from pure India ink of the same concentration) after laser irradiation is presumably from the rapid, laser-activated vaporization of INDs. As laser excitation continued, the supply of vaporizing INDs was depleted, causing the PA signal to drop down to a steady state at a lower level, which is determined by the conventional photoacoustic effect of the encapsulated ink particles. Our speculation can be confirmed by the PA signal of the same concentration's India ink solution, as shown in Fig. 4B, which has PA amplitude at the same level as that of laser excited INDs upon reaching the steady state. Fig. 4C shows the PA maximal amplitude projection (MAP) images of $5,10,20,40 \mathrm{mg} / \mathrm{mL}$ INDs in $0.2 \%$ agarose gel when illuminated with $0.5 \mathrm{~mJ} / \mathrm{cm}^{2}$ laser fluence (800 nm, OPO laser). The PA signal originated from the steady state thermoelastic expansion of the ink particles encapsulated in the INDs. Fig. 4D is the quantitative analysis of the signal intensity over concentration in Fig. 4C, which shows that the PA am- plitude increased linearly with higher INDs concentrations $(\mathrm{P}<0.05)$.

Fig. 5A shows the US images of INDs, pure ink (same ink particle concentration as that incorporated in INDs), and pure droplet (without any ink incorporated, same PLGA concentration as INDs) solutions before and after laser irradiation with a fluence of 5 $\mathrm{mJ} / \mathrm{cm}^{2}$ for $10 \mathrm{~s}$ (532 nm, Nd:YAG laser), while Fig. 5B is the statistical/quantitative analysis of these images. In each image of Fig. 5A, there are two counterparts, with each corresponding to the commercial US system's routine mode (in gray scale) and contrast mode (in color/golden scale) imaging, respectively. The US signals of the routine mode image originated from both INDs and the vaporized bubbles while the signals of the contrast mode image originated solely from the vaporized bubbles. Therefore the contrast mode images were used for all subsequent quantitative analyses of the INDs vaporization. The post irradiation US signal enhancement in the INDs group is much larger than that of the other two groups (also validated by the quantitative analysis in Fig. 5B), where no significant enhancement is observed.

\section{In vivo INDs imaging}

Fig. 6A shows in vivo Bscan PA images of the tumor area before and after intratumoral injection of $40 \mathrm{mg} / \mathrm{mL}$ INDs, pure ink (same ink particle concentration as that incorporated in INDs), and pure droplet (without any ink incorporated, same PLGA concentration as INDs). It is to be noted that, due to the laser power limitation of our PA imaging system (800 $\mathrm{nm}$, OPO laser, laser fluence is $\left.<1 \mathrm{~mJ} / \mathrm{cm}^{2}\right)$, no phase-transition related PA signals were able to be excited during in vivo INDs imaging in this study, i.e., the PA signal enhancement after intratumoral injection derived primarily from the thermoelastic expansion of the encapsulated ink particles. It can be seen that the PA signals increased significantly after the intratumoral injection of INDs and pure ink, sug- 
gesting that, even without triggering the phase-transition, the INDs can still be used for contrast-enhanced PA imaging. Fig. 6B is the quantitative analysis of the images in Fig. 6A, which shows the
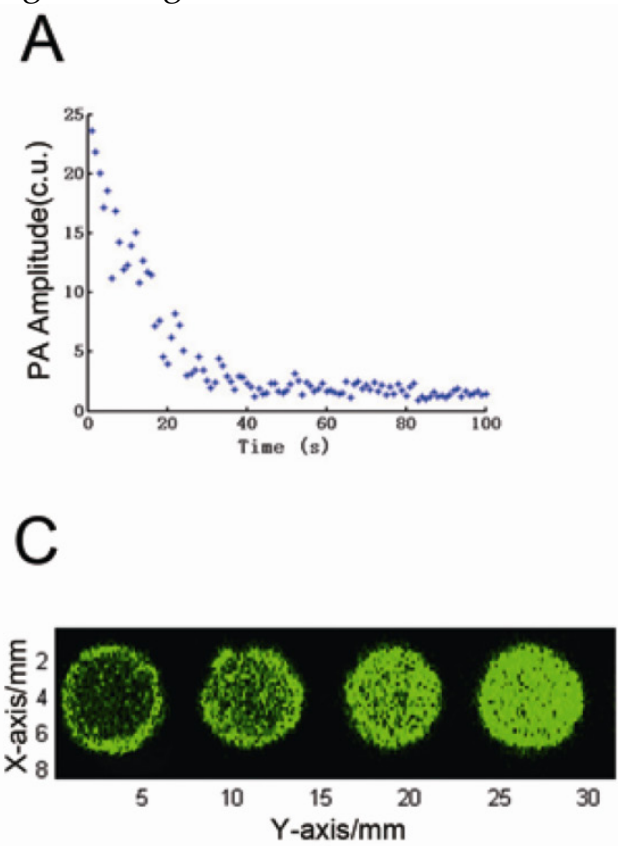

Figure 4. A and B, PA signal dynamics of INDs and ink solution, respectively, during pulsed laser irradiation for $100 \mathrm{~s}\left(800 \mathrm{~nm}, \mathrm{OPO}\right.$ laser, laser fluence is $\left.8 \mathrm{~mJ} / \mathrm{cm}^{2}\right)$. C, The PA maximal amplitude projection (MAP) images of $5,10,20,40 \mathrm{mg} / \mathrm{mL}$ INDs in $0.2 \%$ agarose gel when illuminated with a laser fluence of $0.5 \mathrm{~mJ} / \mathrm{cm}^{2}(800 \mathrm{~nm}, \mathrm{OPO}$ laser). D, The quantitative analysis of the signal intensity over concentration in $\mathrm{C}$.

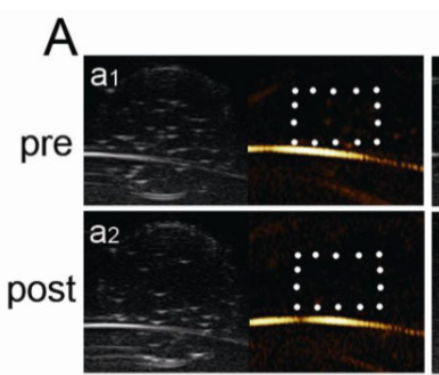

pure droplets

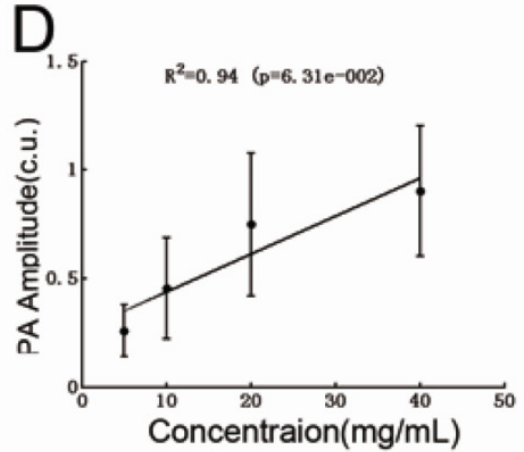

post injection amplitude is $\sim 20$-fold higher than that of the pre-injection case $\left({ }^{*} \mathrm{P}<0.05\right)$ in both INDs and pure ink group.

B

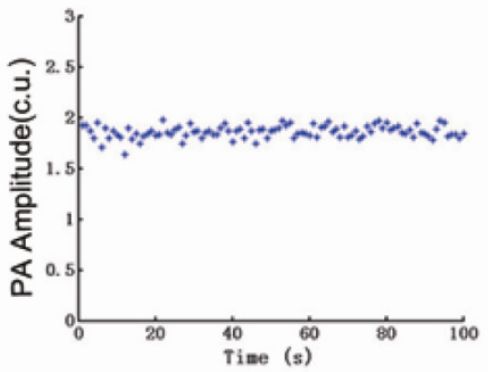

B
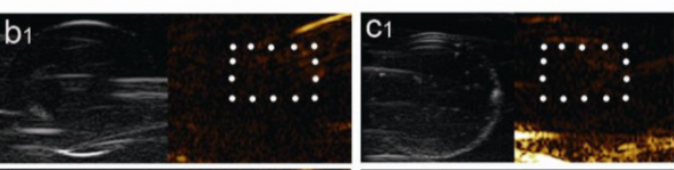

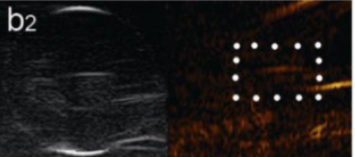

ink

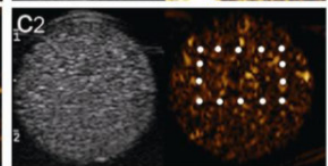

INDs

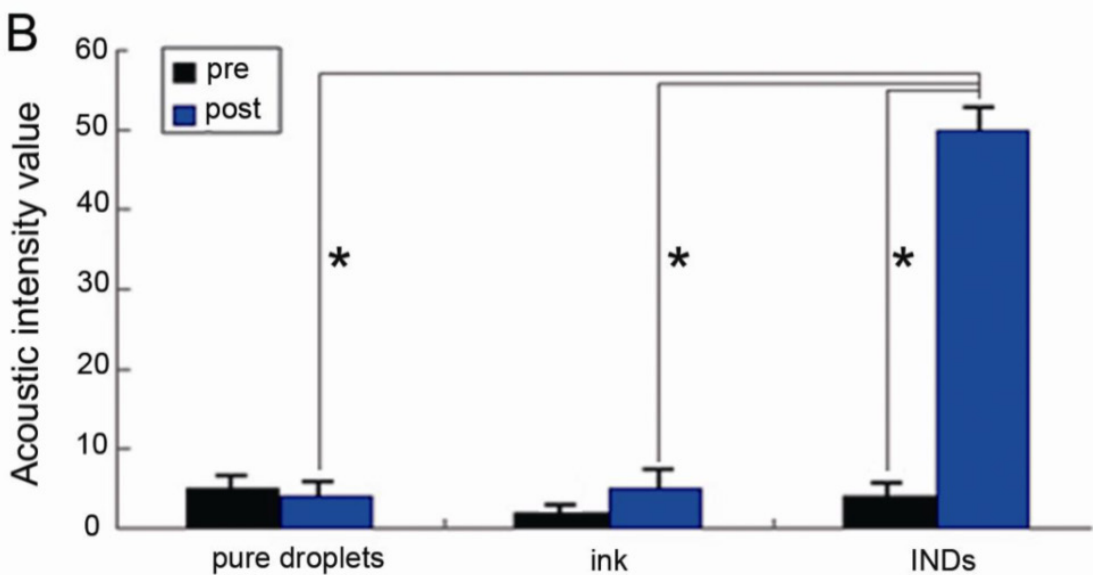

Figure 5. A, US images in routine (gray scale) and contrast (color scale) modes of the INDs, pure ink (same ink particle concentration as that incorporated in INDs), and pure droplets (without any ink incorporated; with the same PLGA concentration as in INDs) solutions before and after laser irradiation with a fluence of $5 \mathrm{~mJ} / \mathrm{cm}^{2} \mathrm{for} 10 \mathrm{~s}$ ( $532 \mathrm{~nm}$, Nd:YAG laser). B, Quantitative analysis of the images (within white box area) in $A(* P<0.05)$. 


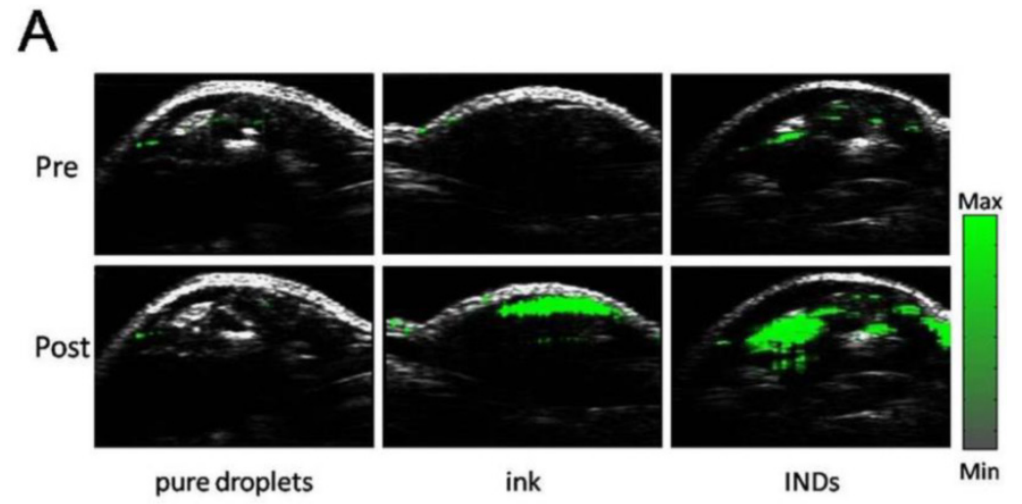

B

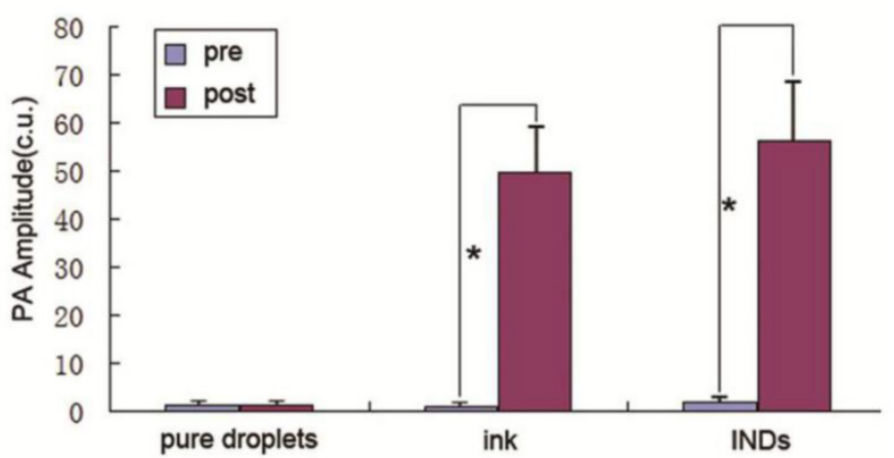

Figure 6. A, In vivo Bscan PA images of the tumor area before and after intratumoral injection of 40 $\mathrm{mg} / \mathrm{mL}$ INDs, pure ink (same ink particle concentration as that incorporated in INDs), and pure droplets (without any ink incorporated, same PLGA concentration as INDs). B, Quantitative analysis of the images intensity in $\mathrm{A}(* \mathrm{P}<0.05)$.

Fig. 7A shows the in vivo US imaging (in both routine and contrast modes) results of the tumor slice after intratumoral injection of INDs, pure ink (same ink particle concentration as that incorporated in INDs), and pure droplet (without any ink incorporated, same PLGA concentration as INDs) before and after laser irradiation with a laser fluence of 12 $\mathrm{mJ} / \mathrm{cm}^{2}$ for $10 \mathrm{~s}$ (532 nm, Nd:YAG laser). Fig. 7B is the quantitative analysis of these images. The same as in phantoms, the US signal enhancement post laser irradiation in the INDs group (20 times enhancement with $40 \mathrm{mg} / \mathrm{mL}$ INDs as used in this study; 10 times and 4 times enhancement can also be achieved with 20 $\mathrm{mg} / \mathrm{mL}$ and $10 \mathrm{mg} / \mathrm{mL}$ INDs, respectively) is much higher than that of the other two groups (also validated by the quantitative analysis in Fig. 7B), where no significant enhancement is observed. It is to be noted that due to the increased optical absorption and scattering in vivo, the energy density needed for triggering the vaporization of INDs is higher than that required in vitro $\left(12 \mathrm{~mJ} / \mathrm{cm}^{2}\right.$ vs. $\left.5 \mathrm{~mJ} / \mathrm{cm}^{2}\right)$. However, this energy level is still fairly low (for example, compared with the energy level needed for photothermal therapy ${ }^{47}$ ) and well below the ANSI laser safety limit. ${ }^{43}$ Therefore, our results showed that in vivo vaporization of INDs can be triggered with low laser energy and short irradiation time. The generated gaseous bubbles generate a significant acoustic impedance mismatch with the surrounding environment, providing excellent contrast for US imaging in vivo.

\section{Impacts of INDs after laser exposure}

No obvious coagulative necrosis was observed based on macroscopic inspections after PA imaging (Fig. 8a $\mathrm{a}_{1}$ ), US imaging (Fig. $8 b_{1}$ ), and in the control group of tumor therapy study (Fig. 8d ${ }_{1}$ ). Neither did HE staining of tumor slices show obvious tis-

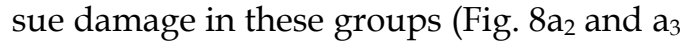
for PA imaging; Fig. $8 b_{2}$ and $b_{3}$ for US imaging; Fig. $8 \mathrm{~d}_{2}$ and $\mathrm{d}_{3}$ for the control group of tumor therapy study), and the tumor cells were intact arranged in nests before and after laser exposure. However, for the INDs + laser group in the tumor therapy study, when the laser fluence reached $\sim 18$ $\mathrm{mJ} / \mathrm{cm}^{2}$ with an exposure duration of $3 \mathrm{~min}$ (532 nm, Nd:YAG laser), the coagulative necrosis was observed (Fig. 8$c_{1}$ ); massive cell injuries were also seen by HE staining, featured with fragments of cell membranes and nuclei in the necrotic area of homogeneous red staining (Fig. $8 \mathrm{c}_{2}$ and $\mathrm{c}_{3}$ ). Similarly, the expression of apoptosis-positive cells based on immunohistochemical examination showed significant tumor apoptosis in the INDs + laser group of tumor therapy study, which was not seen in the control group and the PA and US imaging groups (Fig. 9d vs. Fig. 9b, 9a, 9c). The apoptosis index (AI) in the INDs + laser group was also much higher than that of the other three groups (Fig. 9B). The TEM results showed that, in the control group of the tumor therapy study, as well as the PA and US imaging groups (Fig. 10A-C), both cell and nucleus membranes were intact, although local mitochondria were slightly distended. However, in the INDs + laser group, most cell membranes were incomplete, and many organelles disappeared (Fig. 10D). The images also confirmed that ink particles were present within the cytoplasm or endocytosed cell membranes (Fig. 10E and F). The surface temperature of the tumors in all imaging and therapy groups was measured with an infrared thermometer before and during laser exposure and it showed that there was only a slight temperature rise (within $2^{\circ} \mathrm{C}$, Figure 11) for all the mice. These results revealed that the INDs was suitable for diagnostic PA and US imaging without producing severe thermal damage to the tissue with the laser fluence used in this study. Furthermore, for the laser + INDs 
group, obvious tumor tissue damages were observed by macroscopic, TEM, and pathological inspections, which indicated that the tumor damage was the result of the photoacoustic effect due to the combined use of INDs and laser exposure, rather than the photothermal effect (as no obvious temperature increase was detected). More specifically, the PA therapy mechanism was speculated to mainly originate from the shock-wave generation due to the strong ink (carbon black) particle absorption of the delivered laser energy, rather than the vaporization process of the $\mathrm{PFH}$ droplet. In addition, this speculation and the above experimental results are also consistent with those reported in the literature. ${ }^{36,37,48}$

\section{Conclusions}

In conclusion, using a modified three-step emulsion process, we developed a first-of-its-kind India ink incorporated PFC nanodroplet (IND) material that can be triggered into gas phase upon pulsed laser illumination of low energy. The developed INDs not only provide three types of imaging contrasts - thermoelastic/conventional photoacoustic contrast, phase-transition/nonlinear photoacoustic contrast, and ultrasound contrast-but also offer a potential for cancer therapy through photoacoustic effect induced shock-wave generation from the carbon particles in the ink. Experiments have shown that the developed INDs are chemically stable and spectroscopically robust (ink particles absorb strongly across a wide spectral range from visible to NIR regions), and are of no observed toxicity and immunogenicity. These properties, together with their unique imaging contrasts and therapeutic effect, make the INDs a promising candidate for many theranostic biomedical applications, including photoacoustic effect mediated cancer therapy in conjunction with photoacoustic and ultrasound dual-modality molecular imaging for therapy guidance, monitoring, and evaluation. In addition, as demonstrated in previous works, ${ }^{49,50}$ the INDs may be surface or internally modified to permit molecular targeting of specific biological targets, as well as delivering therapeutic drugs, to enable even more potent multi-functional theranostic applications.
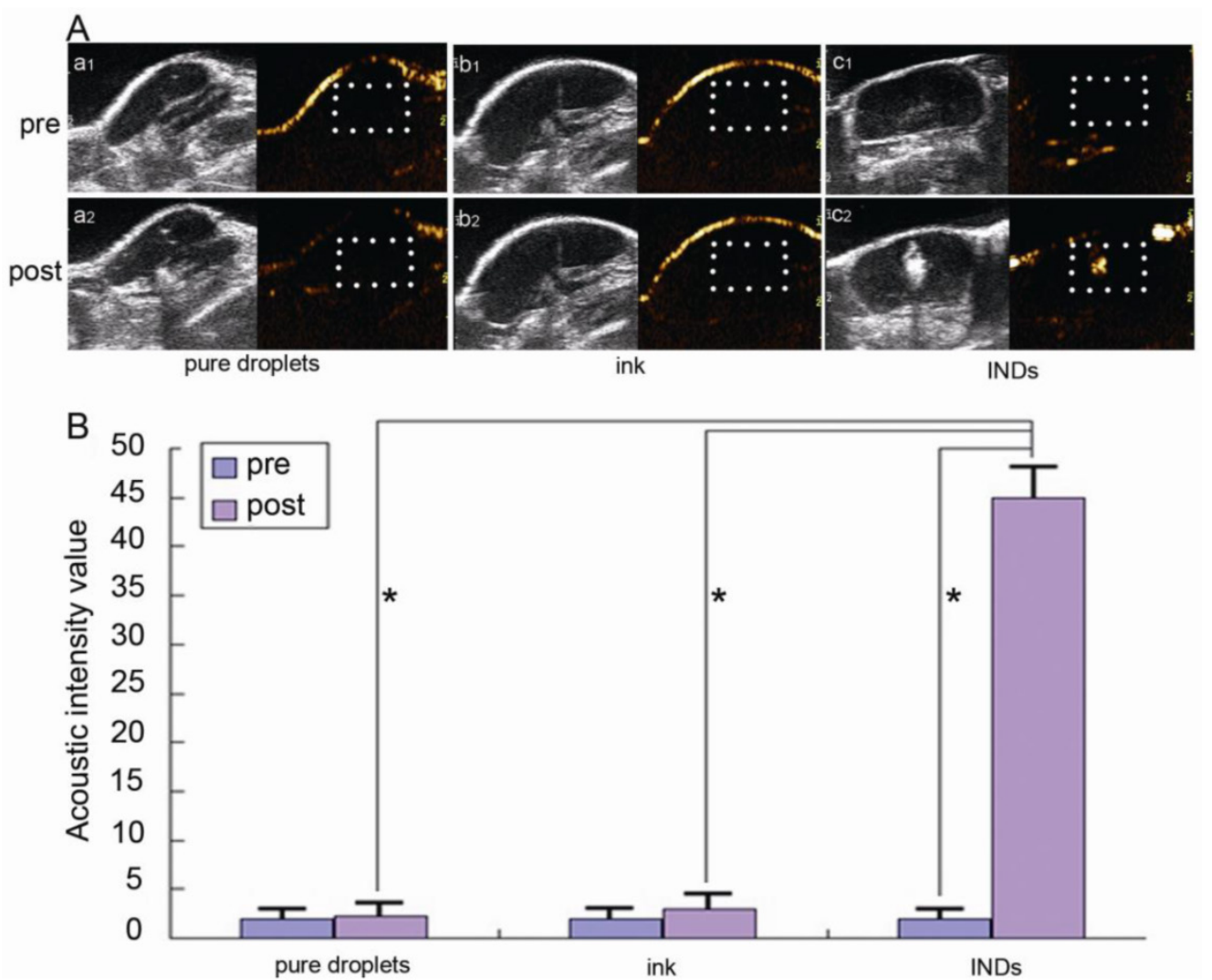

Figure 7. A, In vivo US images in both routine (gray scale) and contrast (color scale) modes of the tumor slice after intratumoral injection of INDs, pure ink (same ink particle concentration as that incorporated in INDs), and pure droplets (without any ink incorporated, same PLGA concentration as INDs) before and after laser irradiation with a fluence of $12 \mathrm{~mJ} / \mathrm{cm}^{2}$ for $10 \mathrm{~s}(532 \mathrm{~nm}, \mathrm{Nd}$ :YAG laser). B, Quantitative analysis of the images (within white box area) in A (*P $<0.05)$. 

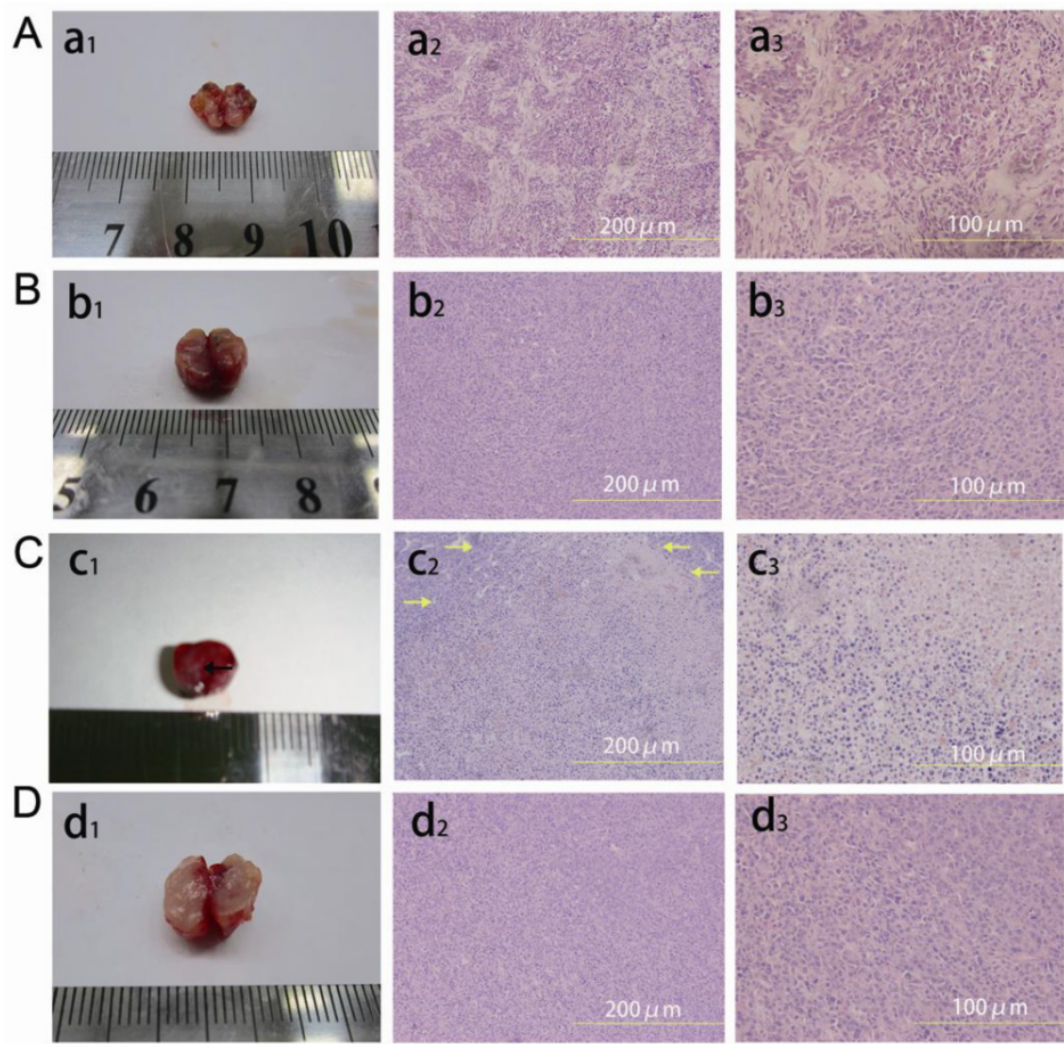

Figure 8. Macroscopic inspection $\left(a_{1}, b_{1}, c_{1}, d_{1}\right)$ and $H E$ staining of tumor tissues at different magnifications $\left(\times 200\left(a_{2}, b_{2}, c_{2}, d_{2}\right)\right.$ and $\left.\times 400\left(a_{3}, b_{3}, c_{3}, d_{3}\right)\right)$ after laser exposure. $A, a_{1}, a_{2}, a_{3}$ are tumor tissues after PA imaging; $B, b_{1}, b_{2}, b_{3}$ are tumor tissues after US imaging; $\mathrm{C}, \mathrm{C}_{1}, \mathrm{C}_{2}, \mathrm{C}_{3}$ are tumor tissues after laser irradiation in the INDs + laser group of the therapy study; D, $d_{1}$, $d_{2}, d_{3}$ are tumor tissues after laser irradiation in the control group of the therapy study. Coagulative necrosis was seen in $\mathrm{c}_{\mathrm{I}}$ (indicated by a black arrow), and necrosis areas were indicated by yellow arrows in $\mathrm{c}_{2}$.

Figure 9. A, Immunohistochemical examination of the tumor tissues after PA imaging (a), laser irradiation in the control group of the therapy study (b), US imaging (c), and laser irradiation in the INDs + laser group of the therapy study (d). Representative apoptosis-positive cells were indicated by black arrows while the apoptosis-negative cells were indicated by yellow arrows. B, The calculated apoptosis index of different groups after laser exposure $(* \mathrm{P}<0.05)$.

A
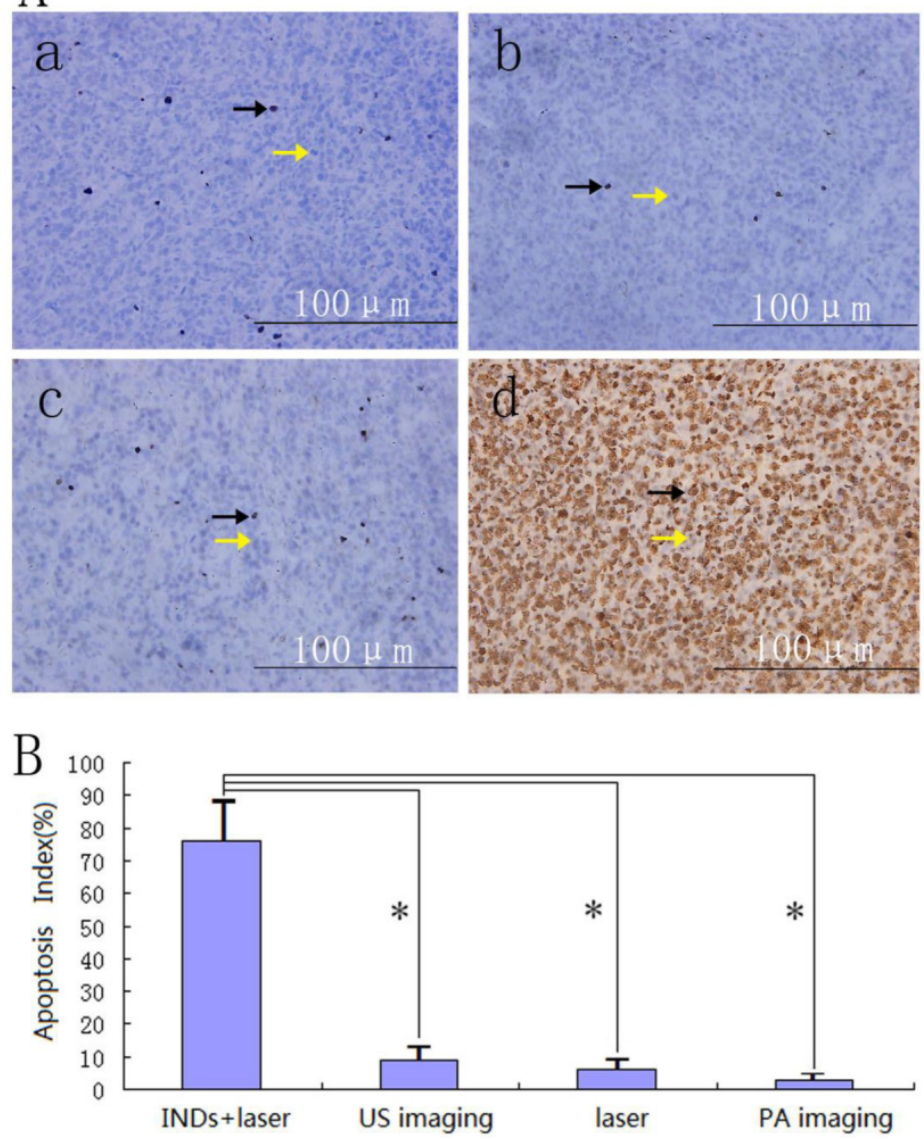

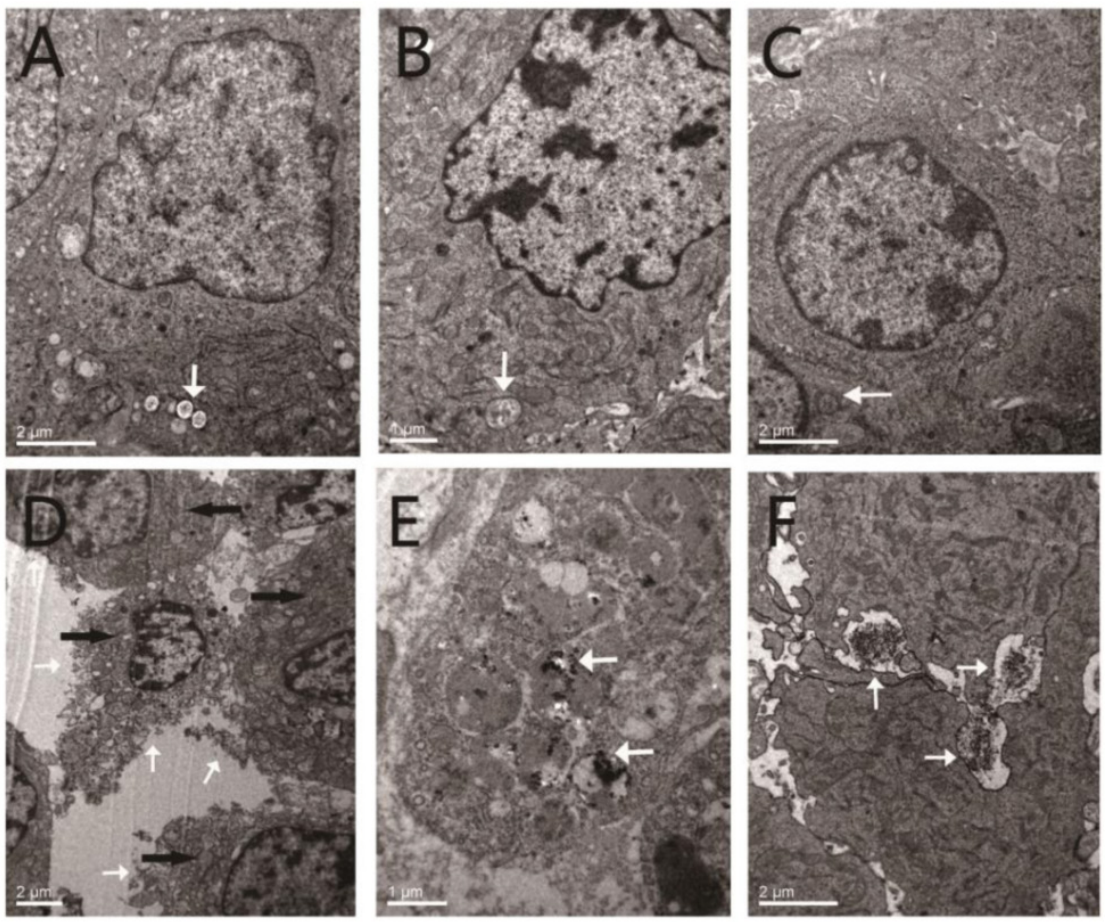

Figure 10. A-C, TEM images of the tumor tissues after laser irradiation in the control group of the therapy study, PA imaging, and US imaging, respectively. The white arrows indicate the slightly distended local mitochondria. D-E, TEM images of the tumor tissues after laser irradiation in the INDs + laser group of the therapy study. F, TEM image of the tumor tissues after laser irradiation in the US imaging group. The white arrows in D indicate the incomplete (damaged) cell membranes, and the black arrows indicate the locations of disappeared cell organelles. The white arrows in E and $F$ indicate the ink particles within either the cytoplasm (E) or endocytosed cell membranes (F).

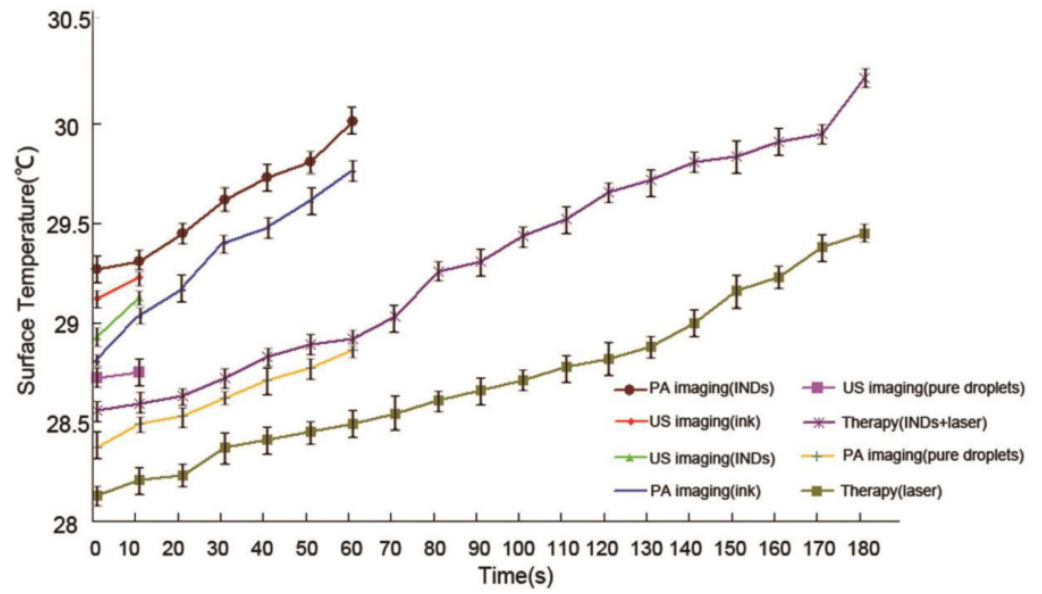

Figure I I. The surface temperature rise of the tumors for all the mice in the imaging and therapy groups.

\section{Acknowledgments}

The authors are grateful to Dr. Zipeng He, Jiaxiang Zheng, Jianhua Chen, and Riqiang Lin for their assistance with PA and US imaging. This work was supported in part by the National Natural Science Foundation of China Grants: 30770565, 81227801, 81161120548, 81130025, 81371579 and 61205203; the National Key Basic Research (973) Program of China: 2014CB744503; the International S\&T Cooperation Program of China: 2014DFG32800; the Shenzhen Science and Technology Innovation Committee grants: ZDSY20130401165820357, KQCX20120816155844962, CXZZ20120617113635699, and JCYJ20120615125857842; and the fund of Chongqing university scientific innovation team 2013(KJTD201303).

\section{Competing Interests}

The authors have declared that no competing interest exists.

\section{References}

1. Sun $Y$, Zheng $Y$, Ran H, et al. Superparamagnetic PLGA-iron oxide microcapsules for dual-modality US/MR imaging and high intensity focused US breast cancer ablation. Biomaterials. 2012; 33: 5854-64.

2. Niu C, Wang Z, Zuo G, et al. Poly(lactide-co-glycolide) ultrasonographic microbubbles carrying sudan black for preoperative and intraoperative localization of lymph nodes. Clin Breast Cancer. 2012; 12: 199-206.

3. Oeffinger BE, Wheatley MA. Development and characterization of a nano-scale control agent. Ultrasonics. 2004; 42: 343-7. 
4. Zhang J, Coulston RJ, Jones ST, et al. One-step fabrication of supramolecular microcapsules from microfluidic droplets. Science. 2012; 335: 690-4.

5. Correas JM, Quay SD. EchoGen emulsion: a new ultrasound contrast agent based on phase shift colloids. Clin Radiol. 1996; 51: 11-4.

6. Kripfgans OD, Fowlkes JB, Miller DL, et al. Acoustic droplet vaporization for therapeutic and diagnostic applications. Ultrasound Med Biol. 2000; 26: 1177-89.

7. Couture $\mathrm{O}$, Bevan $\mathrm{PD}$, Cherin E, et al. A model for reflectivity enhancement due to surface bound submicrometer particles. Ultrasound Med Biol. 2006; 32: 1247-55.

8. Singh R, Husseini GA, Pitt WG. Phase transitions of nanoemulsions using ultrasound: experimental observations. Ultrason Sonochem. 2012; 19: 1120-5.

9. Sheeran PS, Luois S, Dayton PA, et al. Formulation and acoustic studies of a new phase-shift agent for diagnostic and therapeutic ultrasound. Langmuir. 2011; 27: 10412-20.

10. Strohm E, Rui M, Gorelikov I, et al. Vaporization of perfluorocarbon droplets using optical irradiation. Biomed Opt Express. 2011; 2: 1432-42.

11. Wilson K, Homan K, Emelianov S. Biomedical photoacoustics beyond thermal expansion using triggered nanodroplet vaporization for contrast-enhanced imaging. Nat Commun. 2012; 3: 618.

12. Bunkin FV, Komissatov VM. Optical excitation of sound waves. Soviet Physics Acoustics. 1973; 19: 203-11

13. Bunkin FV, Tribel'skii MI. Nonresonant interaction of high-power optical radiation with a liquid. Soviet Physics Uspekhi. 1980; 23: 105.

14. Lyamshev LM, Naugol'nykh KA. Optical generation of sound: nonlinear effects. Soviet Physics Acoustics. 1981; 27: 641-68

15. Strohm E, Rui M, Gorelikov I, et al. Optical droplet vaporization of micron-sized perfluorocarbon droplets and their photoacoustic detection. Proc SPIE. 2011; 7899: 78993H-7.

16. Wang LV, Hu S. Photoacoustic tomography: in vivo imaging from organelles to organs. Science. 2012; 335: 1458-62.

17. Wang LV. Multiscale photoacoustic microscopy and computed tomography. Nat Photonics. 2009; 3: 503-9.

18. Li Z, Yin S, Liu Z, et al. Magnetic targeting enhanced theranostic strategy based on multimodal imaging for selective ablation of cancer. Adv. Funct. Mater. 2014; 24: 2312-21.

19. Song X, Gong H, Liu Z, et al. Ultra-small iron oxide doped polypyrrole nanoparticles for in vivo multimodal imaging guided photothermal therapy. Adv. Funct. Mater. 2014; 24: 1194-201.

20. Kim C, Qin R, Xu JS, et al. Multifunctional microbubbles and nanobubbles for photoacoustic and ultrasound imaging. J. Biomed. Opt. 2010; 15: 010510.

21. Jeon M, Wang S, Huynh E, et al. Methylene blue microbubbles as a model dual-modality contrast agent for ultrasound and activatable photoacoustic imaging. J. Biomed. Opt. 2014; 19: 16005.

22. Huynh E, Lovell JF, Helfield BL, et al. Porphyrin shell microbubbles with intrinsic ultrasound and photoacoustic porperties. J. Am. Chem. Soc. 2012; 134: 16464-7.

23. Song L, Wang LV. Photoacoustic tomography and its applications in drug delivery and photothermal therapy. In drug delivery applications of noninvasive imaging: validation from biodistribution to sites of action; Li C, Tian M, Eds, New Jersey: Wiley; 2013: 45-69.

24. Luke GP, Yeager D, Emelianov SY. Biomedical applications of photoacoustic imaging with exogenous contrast agents. Ann Biomed Eng. 2011; 40: 422-37.

25. Wei C, Lombardo M, Smith KL, et al. Nonlinear contrast enhancement in photoacoustic molecular imaging with gold nanosphere encapsulated nanoemulsions. Appl Phys Lett. 2014; 104: 033701-4.

26. Hannah A, Luke G, Wilson K, et al. Indocyanine green-loaded photoacoustic nanodroplets: dual Contrast nanoconstructs for enhanced photoacoustic and ultrasound imaging. ACS Nano. 2014; 8: 250-9.

27. Zhao B, Itkis ME, Niyogi S, et al. Study of the extinction coefficients of single-walled carbon nanotubes and related carbon materials. J. Phys. Chem. B. 2004; 108: 8136-41.

28. Landsman J, Kwant G, Mook A, et al. Light- absorbing properties, stability, and spectral stabilization of indocyanine green. J. Appl. Physiol. 1976; 40: 575-583.

29. Ninni PD, Martelli F, Zaccanti G. The use of india ink in tissue-simulating phantoms. Opt Express. 2010; 18: 26854-65.

30. Goda F, O'Hara JA, Rhodes ES, et al. Changes of oxygen tension in experimental tumors after a single dose of X-ray irradiation. Cancer Res. 1995; 55: 2249-52.

31. Goda F, Liu KJ, Walczak T, et al. In vivo oximetry using EPR and india ink. Magn Reson Med. 1995; 33: 237-45.

32. Williams BB, Khan N, Zaki B, et al. Clinical electron paramagnetic resonance (EPR) oximetry using india ink. In oxygen transport to tissue XXXI; Takahashi, Eiji, Bruley, Duane F, Eds, Berlin: Springer; 2010: 149-56.

33. Swartz HM, Liu KJ, Goda F, et al. India ink: a potential clinically applicable EPR oximetry probe. Magn Reson Med. 1994; 31: 229-32.

34. Zerda DL, Zavaleta AC, Keren S, et al. Carbon nanotubes as photoacoustic molecular imaging agents in living mice. Nat Nanotechnol. 2008; 3: 557-62

35. Ajayan PM, Terrones M, Guardia A, et al. Nanotubes in a flash-ignition and reconstruction. Science. 2002; 296: 705

36. Kang B, Dai Y, Chang S, et al. Explosion of single-walled carbon nanotubes in suspension induced by a large photoacoustic effect. Carbon. 2008; 46: 978-81.

37. Kang B, Yu D, Dai Y, et al. Cancer-cell targeting and photoacoustic therapy using carbon nanotubes as "bomb" agents. Small. 2009; 5: 1292-301.
38. Schutt EG, Klein DH, Mattrey RM, et al. Injectable microbubbles as contrast agents for diagnostic ultrasound imaging: the key role of perfluorochemicals . Angew Chem Int Ed. 2003; 42: 3218-35.

39. Jain RA. The manufacturing techniques of various drug loaded biodegradable poly (lactide-co-glycolide) (PLGA) devices. Biomaterials. 2000; 21: 2475-90.

40. Rosa GD, Quaglia F, Rotonda MIL, et al. Biodegradable microparticles for the controlled delivery of oligonucleotides. Int J Pharm. 2002; 242: 225-8.

41. Zheng C, Zheng M, Gong P, et al. Indocyanine green-loaded biodegradable tumor targeting nanoprobes for in vitro and in vivo imaging. Biomaterials. 2012; 33: 5603-9.

42. Sheng Z, Song L, Zheng J, et al. Protein-assisted fabrication of nano-reduced graphene oxide for combined in vivo photoacoustic imaging and photothermal therapy. Biomaterials. 2013; 34: 5236-43.

43. ANSI Orlando: Laser Institute of America. American National Standard for Safe Use of Lasers, ANSI Z136.1 (American National Standards Institute, New York, 2000).

44. Ranganath $\mathrm{SH}, \mathrm{Fu} \mathrm{Y}$, Arifin DY, et al. The use of submicron /nanoscale PLGA implants to deliver paclitaxel with enhanced pharmacokinetics and therapeutic efficacy in intracranial glioblastoma in mice. Biomaterials. 2010; 31: 5199-207.

45. Hiltbrand E, Belenger J, Binzoni T, et al. A new method of thermoablation with hot water vapour for localized tumours. Anticancer Res. 2004; 24: 2757-63.

46. Danhier F, Lecouturier N, Vroman B, et al. Paclitaxel-loaded PEGylated PLGA-based nanoparticles: in vitro and in vivo evaluation. J Controlled Release. 2009; 133: 11-7.

47. Zheng X, Zhou F, Wu B, et al. Enhanced tumor treatment using biofunctional indocyanine green-containing nanostructure by intratumoral or intravenous injection. Mol Pharm. 2012; 9: 514-22.

48. Zhong J, Yang S, Zheng X, et al. In vivo photoacoustic therapy with cancer-targeted indocyanine green-containing nanoparticles. Nanomedicine. 2013; 8: 903-19.

49. Klibanov AL. Preparation of targeted microbubbles: ultrasound contrast agents for molecular imaging. Med Biol Eng Comput. 2009; 47: 875-82.

50. Kaneda MM, Caruthers S, Lanza GM, et al. Perfluorocarbon nanoemulsions for quantitative molecular imaging and targeted therapeutics. Ann Biomed Eng. 2009; 37: 1922-33. 